\title{
Electronic Structure in the Bulk State of $\mathrm{SrTiO}_{3-\delta}$ by Soft-X-Ray Spectroscopy
}

\author{
Tohru Higuchi, Naoko Kato and Yuko Suzuki \\ Department of Applied Physics, Tokyo University of Science, 1-3 Kagurazaka, Shinjuku, Tokyo 162-8601, Japan \\ Fax: 81-3-5228-8241, e-mail: higuchi@rs.kagu.tus.ac.jp
}

\begin{abstract}
The electronic structure in the bulk state of $\mathrm{SrTiO}_{3-\delta}$ single crystal has studied by X-ray absorption spectroscopy (XAS) and soft-X-ray emission spectroscopy (SXES). The $\mathrm{SrTiO}_{3-\delta}$ exhibits the electrical conductivity in semiconducting region. The Ti $3 d$ DOS is created in the band gap energy region by substituting oxygen vacancies. The SXES spectra exhibit the soft-X-ray Raman scattering structures, which correspond to $d$ - $d$ transition from occupied Ti $3 d$ state to unoccupied Ti $3 d$ state. The peak position of the Raman scattering reflects the half on-site Coulomb energy $\left(U_{d d} / 2\right)$.
\end{abstract}

Keywords: $\mathrm{SrTiO}_{3-\delta}$, soft-X-ray emission spectroscopy (SXES), $d-d$ transition, on-site Coulomb energy

\section{INTRODUCTION}

Perovskite-type oxide $\mathrm{SrTiO}_{3}$ with a band gap of 3.2 $\mathrm{eV}$ exhibits $n$-type conductivity due to doping with electric carriers and becomes a superconductor at low temperature. Such electron doping is known to transform insulating $\mathrm{SrTiO}_{3}$ readily into a metallic state even with a very small extent of doping [1-4]. Doping can be achieved by altering any of the three sublattices, namely, those of $\mathrm{Sr}^{2+}, \mathrm{Ti}^{4+}$ and $\mathrm{O}^{2-}$. In particular, $\mathrm{La}^{3+}$ substitution at the $\mathrm{Sr}^{2+}$ site $\left(\mathrm{Sr}_{1-x} \mathrm{La}_{x} \mathrm{TiO}_{3}\right)$ and $\mathrm{Nb}^{5+}$ substitution at the $\mathrm{Ti}^{4+}$ site $\left(\mathrm{SrTi}_{1-x} \mathrm{Nb}_{x} \mathrm{O}_{3}\right)$ have been extensively studied [1-6]. These samples are used as a substrate for the thin film deposition of ferroelectrics and superconductors because of the their similar crystal structures and good lattice matching. On the other hand, $\mathrm{SrTiO}_{3-\delta}$ with oxygen vacancies, which exists as natural ore, might be also expected as substrate for the thin film deposition and other electronic devices. However, details of electronic structure have not been clarified thus far.

In this study, we prepared the $\mathrm{SrTiO}_{3-\delta}(\delta=0,0.02)$ single crystals by floating zone method and measured the electronic structure using X-ray absorption spectroscopy (XAS) and soft-X-ray emission spectroscopy (SXES). The photoemission (PES) spectra were also measured as reference. The SXES spectra reflect the electronic structure of the bulk compared with PES spectra, because the mean free path of a soft-X-ray is very long compared with that of the electron. Furthermore, the Raman scattering observed in the SXES spectra provides useful information about the electronic structure. It is reported that the Raman scattering for $3 d$ transition metal compounds is attributed primarily to the $d-d$ transition between the $3 d$ valence and conduction bands as well as charge transfer (CT) transition from an occupied $\mathrm{O} 2 p$ band to an unoccupied $3 d$ band [7-12]. In particular, The observations of $d-d$ and CT transitions are useful for the study of electronic structure of strongly correlated electron system. Here, we prove electronic structure and electron correlation effect of $\mathrm{SrTiO}_{3-\delta}$ single crystals.

\section{EXPERIMENTAL}

The $\mathrm{SrTiO}_{3-\delta}(\delta=0,0.02)$ samples were prepared by the solid state reaction of $\mathrm{SrCO}_{3}(99.9 \%$, Furuuchi Chemical Co., Ltd.) and $\mathrm{TiO}_{2}(99.99 \%$, Furuuchi Chemical Co., Ltd.) at $1200^{\circ} \mathrm{C}$ for about $12 \mathrm{~h}$, and the single crystals were grown by a floating-zone method using an Xe-arc imaging furnace. The $\mathrm{SrTiO}_{2.98}$ crystal was obtained in an as-grown sample. The $\mathrm{SrTiO}_{3}$ crystal was obtained by annealing the as-grown crystal in $\mathrm{O}_{2}$ atmosphere at $1000^{\circ} \mathrm{C}$. The prepared crystals were characterized by X-ray diffraction. The electrical resistivity was characterized by AC impedance method.

SXES and XAS spectra were measured using a soft-X-ray spectrometer installed at an undulator beamline BL-19B (at the Photon Factory), of the High Energy Accelerator Organization. Synchrotron radiation was monochromatized using a varied line spacing plane grating whose average groove density is 1000 lines $/ \mathrm{mm}$. The incidence angle of the soft-X-ray was selected to be about $75^{\circ}$ in order to avoid the self-absorption effect. The energy resolution was smaller than $0.8 \mathrm{eV}$ at $h v=450 \mathrm{eV}$.

\section{RESULTS AND DISCUSSION}

Figure 1(a) shows the XRD patterns of (200) peak as a function of anneal temperature in $\mathrm{O}_{2}$ atmosphere for $\mathrm{SrTiO}_{3-\delta}$ single crystal. The (200) peak shifts to the lower diffraction angle with increasing anneal temperature. Figure 1(b) shows the lattice constant of $a$-axis as a function of anneal temperature calculated from Fig. 1(a). The lattice constant increases with increasing anneal temperature. The lattice constant of as-grown $\mathrm{SrTiO}_{3-\delta}$ annealed at $1000^{\circ} \mathrm{C}$ accords with that of pure $\mathrm{SrTiO}_{3}$ single crystal [13]. This indicates that the oxygen vacancies of $\mathrm{SrTiO}_{3-\delta}$ are compensated by annealing at $1000^{\circ} \mathrm{C}$ in $\mathrm{O}_{2}$ atmosphere. 

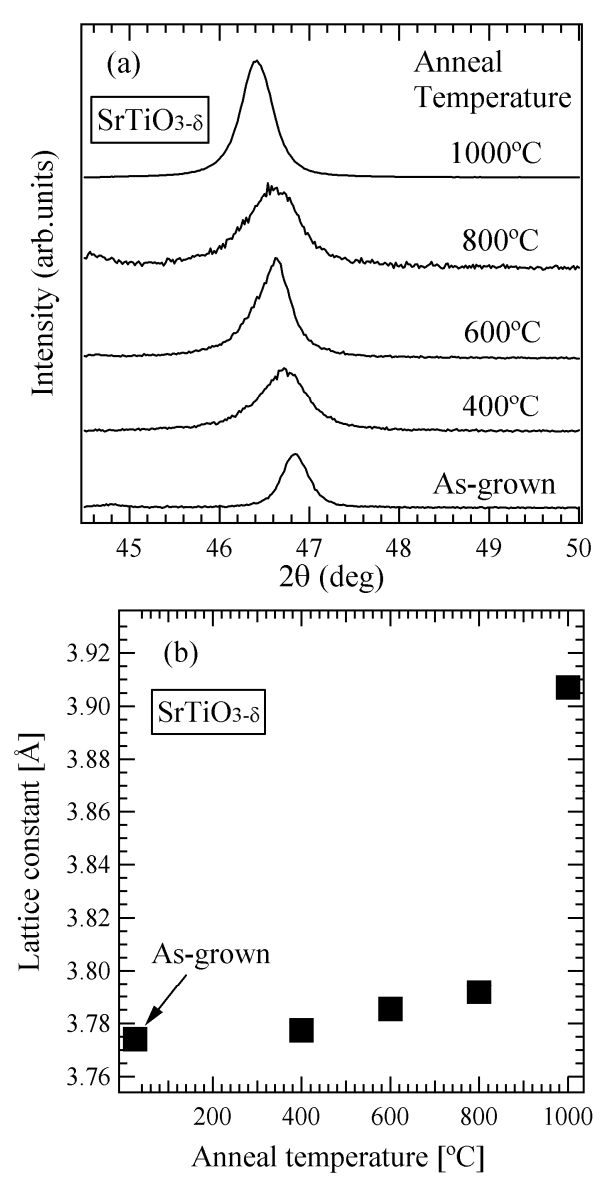

Fig. 1 (a) XRD patterns and (b) lattice constants as a function of anneal temperature in $\mathrm{O}_{2}$ atmosphere.

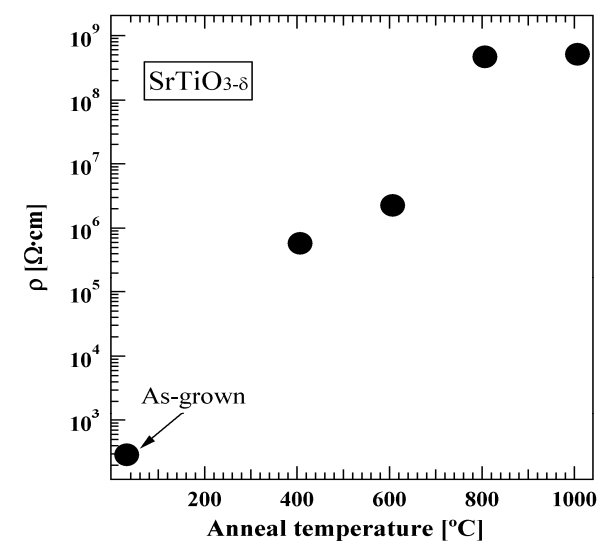

Fig. 2 Electrical resistivity at room temperature as a function of anneal temperature in $\mathrm{O}_{2}$ atmosphere.

Figure 2 shows the electrical resistivity as a function of anneal temperature in $\mathrm{O}_{2}$ atmosphere for $\mathrm{SrTiO}_{3-\delta}$ single crystal. The electrical resistivity increases with increasing anneal temperature. The resistivity of as-grown $\mathrm{SrTiO}_{3-\delta}$ annealed at $1000^{\circ} \mathrm{C}$ accords with that of pure $\mathrm{SrTiO}_{3}$ single crystal. However, the as-grown $\mathrm{SrTiO}_{3-\delta}$ sample exhibits the resistivity in the semiconducting region. This may indicate that the Ti $3 d$ electron is closely related with the decrease of resistivity.

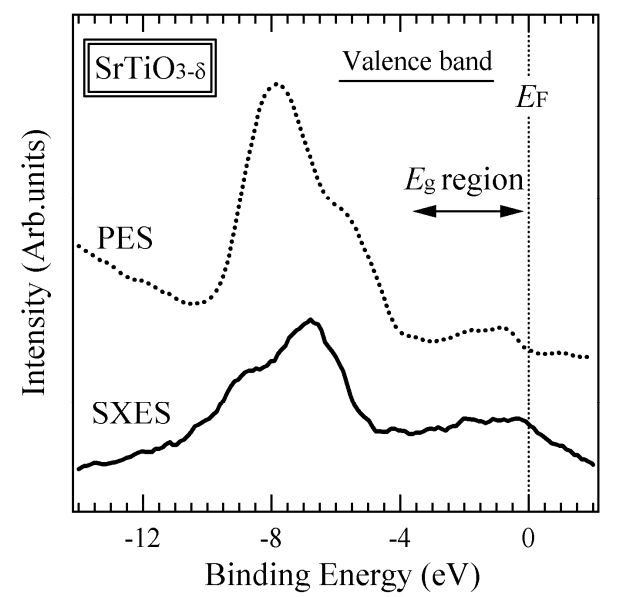

Fig. 3 PES and SXES spectra in the valence band region of as-grown $\mathrm{SrTiO}_{3-\delta}$.

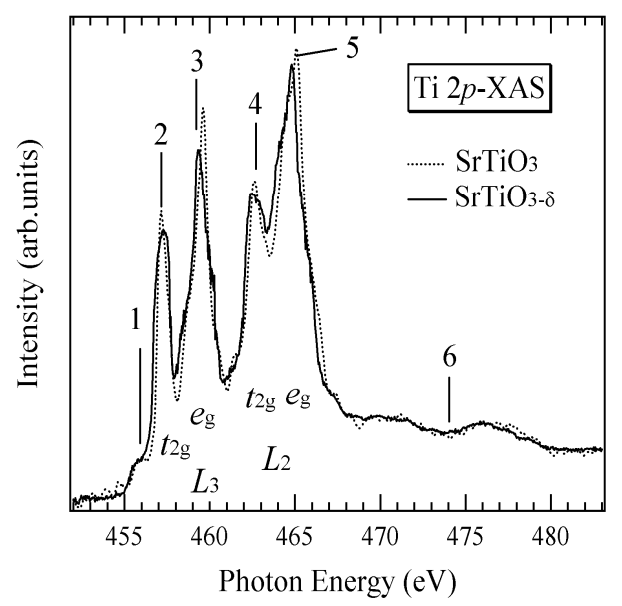

Fig. 4 Ti $2 p$ XAS spectra of $\mathrm{SrTiO}_{3-\delta}$ and pure $\mathrm{SrTiO}_{3}$. The numbers indicate the photon energies where the Ti $2 p$ SXES spectra were measured.

Figure 3 shows the PES and SXES spectra in the valence band region of $\mathrm{SrTiO}_{3-\delta}$. The PES spectrum measured at $h v=450 \mathrm{eV}$ reflects the total density-of-state (DOS). It is well known that the valence band is mainly composed of $\mathrm{O} 2 p$ state hybridized with $\mathrm{Ti} 3 d$ state. Although pure $\mathrm{SrTiO}_{3}$ does not have DOS in the energy gap $\left(E_{\mathrm{g}}\right)$ region, the $\mathrm{SrTiO}_{3-\delta}$ exhibits the existence of DOS. The Ti $2 p$ SXES spectrum measured at $h v=472 \mathrm{eV}$ reflects the Ti $3 d$ partial DOS in the valence band region. The Ti $3 d$ partial DOS is observed in the valence band and $E_{\mathrm{g}}$ regions. In particular, the DOS exists at near Fermi level $\left(E_{\mathrm{F}}\right)$.

Figure 4 shows the Ti $2 p$ XAS spectrum of $\mathrm{SrTiO}_{3-\delta}$ and $\mathrm{SrTiO}_{3}$. The spectrum is mainly derived from two parts of $L_{3}\left(2 p_{3 / 2}\right)$ and $L_{2}\left(2 p_{1 / 2}\right)$. Each part is split into $t_{2 \mathrm{~g}}$ and $e_{\mathrm{g}}$ states by an octahedral ligand field. The spectral shape of $\mathrm{SrTiO}_{3}$ is similar to that of $\mathrm{SrTiO}_{3-\delta}$. The peak intensity of Ti $2 p$ XAS spectrum is lower in $\mathrm{SrTiO}_{3-\delta}$. This indicates that the Ti $3 d$ electron with oxygen vacancies enters into the Ti $3 d$ conduction band. The vertical bars labeled from 1 to 6 indicate photon energies selected for resonant SXES measurements. 


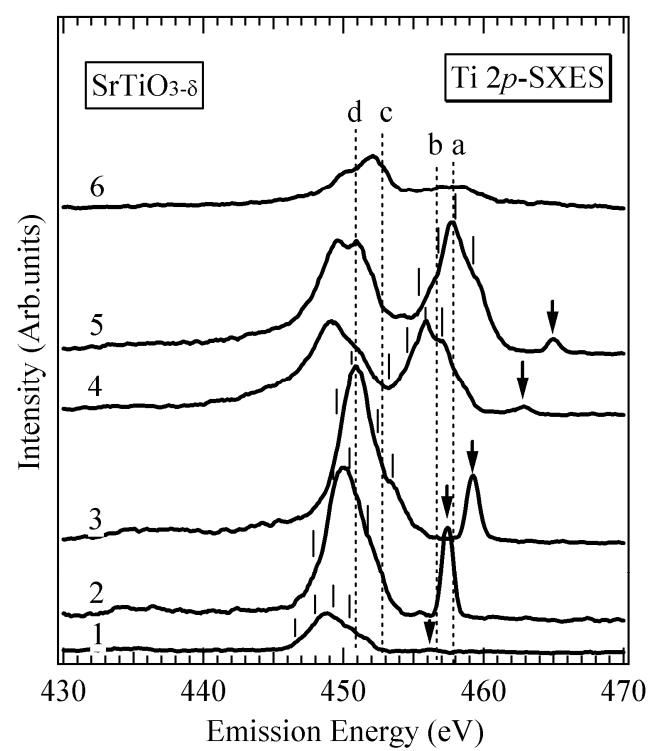

Fig. 5 Ti $2 p$ SXES spectra of $\mathrm{SrTiO}_{2.98}$ excited at various photon energies in Fig. 1. Arrow shows the energy position of the excitations photon energy. Vertical broken lines show the energy positions of Ti $3 d \rightarrow 2 p$ fluorescence.

Figure 5 shows Ti $2 p$ SXES spectra of $\mathrm{SrTiO}_{3-\delta}$ excited at each point of Fig. 1. It is well known that the Ti $2 p$ emission reflects the Ti $3 d$ partial DOS. An arrow shown in each spectrum is attributed to elastic scattering of the excitation photon. The elastic peak is enhanced at the excitation energy corresponding to the $t_{2 \mathrm{~g}}$ absorption peak of $L_{3}$. Then, the peak intensity decreases with increasing excitation energy.

The SXES spectrum 6 excited at $h v=472 \mathrm{eV}$ is an off-resonance spectrum attributed to the normal $\mathrm{Ti}$ $3 d \rightarrow 2 p$ fluorescence spectrum. This spectrum suggests that the Ti $3 d$ state hybridizes with the $\mathrm{O} 2 p$ state in the valence band, as shown in Fig. 3. Four dashed lines (a, $\mathrm{b}, \mathrm{c}$, and d peaks) show the fluorescence bands. The a and $\mathrm{b}$ peaks correspond to the bonding state and the nonbonding state in the valence band, respectively. The $\mathrm{c}$ and $\mathrm{d}$ peaks correspond to the coherent band and the incoherent band in the band gap, respectively.

Four parts denoted by small vertical bars in each $\mathrm{Ti}$ $2 p$ SXES spectrum represent the energy sites shifted from the excitation energy shown in Fig.4, to be 5.6, 6.9, 8.0 , and $9.3 \mathrm{eV}$, respectively. They shift as the excitation energy is varied. These features are attributed to the soft-X-ray Raman scattering (or inelastic scattering). The soft-X-ray Raman scattering that is excited in the $L_{3}$ absorption spectral region overlaps with the Ti $3 d \rightarrow 2 p$ fluorescence. The SXES spectrum 1 excited immediately below the $\mathrm{Ti} 2 p$ threshold shows an apparent feature at a lower energy than the elastic scattering. Since the excitation energy is lower than the binding energy of Ti $2 p$, the Ti $3 d \rightarrow 2 p$ fluorescence cannot be observed. It is attributed to a normal Raman scattering, where the intermediate is a virtual state.

Figure 6 shows SXES spectra of $\mathrm{SrTiO}_{3-\delta}$, where the abscissa is the Raman shift (or energy loss) that is the energy shift from the elastic scattering. The energy of

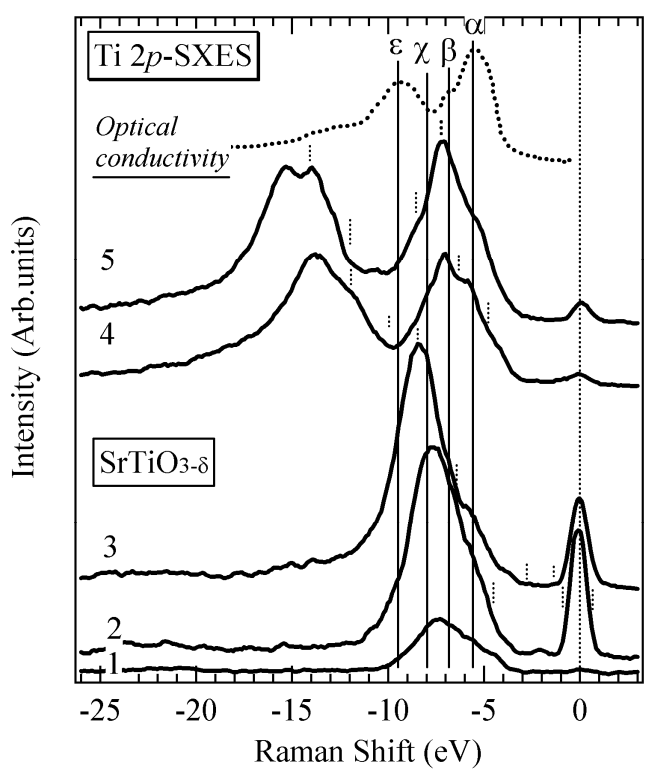

Fig. $6 \mathrm{Ti} 3 d \rightarrow 2 p$ SXES spectra of $\mathrm{SrTiO}_{3-\delta}$ presented as the relative emission energy to the elastic scattering. Inset shows the optical conductivity spectrum of $\mathrm{SrTiO}_{3-\delta}$.

each elastic scattering peak in Fig. 5 is normalized at 0 $\mathrm{eV}$. The Ti $3 d \rightarrow 2 p$ fluorescence peaks shown by four vertical dashed bars shift to the higher energy with increasing the excitation energy. Four vertical solid lines $\alpha, \beta, \chi$, and $\varepsilon$ indicate the Raman scatterings. These Raman scatterings can be compared with the optical conductivity spectrum [3], since the elementary excitation of the Raman scattering is the valence band transition. Therefore, the optical conductivity spectrum of $\mathrm{SrTiO}_{3-\delta}$ is shown as dashed spectral line in inset of Fig. 6. Compared with the SXES spectra, the four Raman scatterings are in good accordance with the optical conductivity spectrum, as shown by the four dashed lines. This fact indicates that these Raman scatterings can be attributed to a charge-transfer (CT) transition from the occupied $\mathrm{O} 2 p$ state to the unoccupied Ti $3 d$ state $[11,12]$.

Figure 7 shows a comparison of the $t_{2 \mathrm{~g}}$-resonanced SXES spectra between $\mathrm{SrTiO}_{3}$ and $\mathrm{SrTiO}_{3-\delta}$. As reference, the $\mathrm{La}_{0.10} \mathrm{Sr}_{0.90} \mathrm{TiO}_{3}$ (LSTO) with the electron correlation effect is also shown in this figure. The $\delta t_{2 \mathrm{~g}}$ peak is observed at about $2.0 \mathrm{eV}$ in $\mathrm{SrTiO}_{3-\delta}$, although the peak is not observed in $\mathrm{SrTiO}_{3}$. The PES spectrum in Fig. 3 indicates that two peaks attributed to Ti $3 d$ states are observed just below the Fermi level $\left(E_{\mathrm{F}}\right)$. The peaks are the coherent band at $E_{\mathrm{F}}$ and the incoherent band at about $1.5 \mathrm{eV}$ below $E_{\mathrm{F}}$. In the $t_{2 \mathrm{~g}}$ excitation, there is typically no large band splitting so that the contribution to the Raman scattering is due to the electron correlation energy $\left(U_{d d}\right)$. The Raman scattering of the $\delta t_{2 \mathrm{~g}}$ peak at approximately $\sim 2.0 \mathrm{eV}$ corresponds to the $d-d$ transition from the occupied incoherent band to the unoccupied coherent band. Therefore, the Raman shift of the $\delta t_{2 \mathrm{~g}}$ peak reflects the effective $U_{d d} / 2$. The magnitude is in good agreement with the results of the PES and inverse-PES studies [12]. On the other hand, the intensity at Raman shift $=0 \mathrm{eV}$ is 


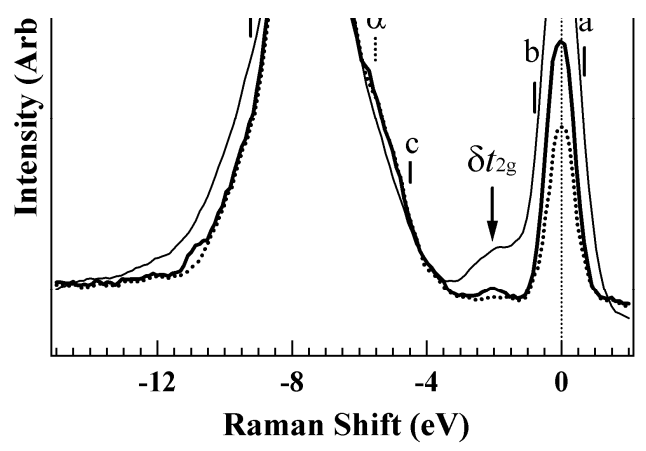

Fig. 7 Comparison of the $t_{2 \mathrm{~g}}$-resonance SXES spectra of $\mathrm{SrTiO}_{3-\delta}$ and pure $\mathrm{SrTiO}_{3}$. This $t_{2 \mathrm{~g}}$-resonance spectrum obtained for $\mathrm{SrTiO}_{3-\delta}$ corresponds to the spectrum 2 in Fig. 6 . As reference, the spectrum of $\mathrm{La}_{0.10} \mathrm{Sr}_{0.90} \mathrm{TiO}_{3}$ (LSTO) is also shown.

larger in $\mathrm{SrTiO}_{3-\delta}$. This behavior indicates the Drude photoresponse which is attributed to the $d-d$ transition between the coherent bands $[9,12]$.

\section{CONCLUSION}

The authors have studied the electronic structure in the bulk state of $\mathrm{SrTiO}_{3-\delta}$ using the SXES, PES and XAS The electronic structure in the valence band region of the SXES spectrum reflects that of the PES spectrum. The CT Raman scattering, which corresponds to the transition from $\mathrm{O} 2 p$ to Ti $3 d$ states in the virtual state, agrees with the optical conductivity spectrum. The Raman scattering, which is attributed to the $d-d$ transition between the incoherent and the coherent bands, is directly observed in the $t_{2 \mathrm{~g}}$-resonance SXES spectra of $\mathrm{SrTiO}_{3-\delta}$. The Raman shift corresponds to the magnitude of the effective $U_{d d} / 2$. The existence of the Raman scattering indicates that the $\mathrm{SrTiO}_{3-\delta}$ has weak electron correlation effect.

\section{ACKNOWLEDGEMENTS}

This work was partly supported by the Grant-in-Aid for Science Research C (No. 22560670) from the Ministry of Education, Culture, Sports, Science and Technology in Japan.
[4] Y. Taguchi, T. Okuda, M. Ohashi, C. Murayama, N. Mohri, Y. Iye and Y. Tokura: Phys. Rev. B 59 (1999) 7917.

[5] T. Higuchi, T. Tsukamoto, N. Sata, M. Ishigame, Y. Tezuka and S. Shin: Phys. Rev. B 57 (1998) 6978.

[6] T. Higuchi, T. Tsukamoto, K. Kobayashi, Y. Ishiwata, M. Fujisawa, T. Yokoya, S. Yamaguchi and S. Shin: Phys. Rev. B 61 (2000) 12860.

[7] S. M. Butorin, J. -H. Guo, M. Magnuson and J. Nordgren: Phys. Rev. B 55 (1997) 4242.

[8] S. Shin, M. Fujisawa, H. Ishii, Y. Harada, M. Watanabe, M. M. Grush, T. A. Callcott, R. C. Perera, E. Z. Kurmaev, A. Moewes, R. Winarski, S. Stadler and D. L. Ederer: J. Electron Spectrosc. Relat. Phenom. 92 (1998) 197.

[9] T. Higuchi, T. Tsukamoto, M. Watanabe, M. M. Grush, T. A. Callcott, R. C. Perera, D. L. Ederer, Y. Tokura, Y. Harada, Y. Tezuka and S. Shin: Phys. Rev. B 60 (1999) 7711.

[10] A. Kotani and S. Shin: Rev. Mod. Phys. 73 (2001) 203.

[11] T. Higuchi, T. Takeuchi, T. Tsukamoto, Y. Harada, Y. Taguchi, Y. Tokura and S. Shin: Nucl. Instrum. \& Methods B 199 (2003) 386.

[12] T. Higuchi, D. Baba, T. Takeuchi, T. Tsukamoto, Y. Taguchi, Y. Tokura, A. Chainani, and S. Shin: Phys. Rev. B 68 (2003) 104420.

[13] V. E. Henrich and R. L. Kortz: J. Vac. Sci. Technol. 18 (1981) 416.

(Received December 25, 2010; Accepted January 14, 2011) 